\title{
BMJ Open Lay health supporters aided by a mobile phone messaging system to improve care of villagers with schizophrenia in Liuyang, China: protocol for a randomised control trial
}

Dong (Roman) Xu, ${ }^{1,2}$ Wenjie Gong, ${ }^{3,4}$ Eric D Caine,${ }^{4}$ Shuiyuan Xiao, ${ }^{3}$ James P Hughes, ${ }^{5}$ Marie Ng, ${ }^{6}$ Jane Simoni, ${ }^{7}$ Hua He, ${ }^{8}$ Kirk L Smith, ${ }^{9}$ Henry Shelton Brown III, ${ }^{10}$ Stephen Gloyd ${ }^{2}$

To cite: $X u D(R)$, Gong W, Caine ED, et al. Lay health supporters aided by a mobile phone messaging system to improve care of villagers with schizophrenia in Liuyang, China: protocol for a randomised control trial. BMJ Open 2016;6:e010120. doi:10.1136/bmjopen-2015010120

- Prepublication history and additional material is available. To view please visit the journal (http://dx.doi.org/ 10.1136/bmjopen-2015010120).

Received 28 September 2015 Revised 10 November 2015 Accepted 2 December 2015

CrossMark

For numbered affiliations see end of article.

Correspondence to Dr Wenjie Gong; gongwenjie@csu.edu.cn

\section{ABSTRACT}

Introduction: Schizophrenia is a severe, chronic and disabling mental illness. Non-adherence to medication and relapse may lead to poorer patient function. This randomised controlled study, under the acronym LEAN (Lay health supporter, e-platform, award, and iNtegration), is designed to improve medication adherence and high relapse among people with schizophrenia in resource poor settings.

Methods/analysis: The community-based LEAN has four parts: (1) Lay health supporters (LHSs), mostly family members who will help supervise patient medication, monitor relapse and side effects, and facilitate access to care, (2) an E-platform to support twoway mobile text and voice messaging to remind patients to take medication; and alert LHSs when patients are non-adherent, (3) an Award system to motivate patients and strengthen LHS support, and (4) iNtegration of the efforts of patients and LHSs with those of village doctors, township mental health administrators and psychiatrists via the e-platform. A random sample of 258 villagers with schizophrenia will be drawn from the schizophrenic ' 686 ' Program registry for the 9 Xiang dialect towns of the Liuyang municipality in China. The sample will be further randomised into a control group and a treatment group of equal sizes, and each group will be followed for 6 months after launch of the intervention. The primary outcome will be medication adherence as measured by pill counts and supplemented by pharmacy records. Other outcomes include symptoms and level of function. Outcomes will be assessed primarily when patients present for medication refill visits scheduled every 2 months over the 6-month follow-up period. Data from the study will be analysed using analysis of covariance for the programme effect and an intent-to-treat approach. Ethics and dissemination: University of Washington: 49464 G; Central South University: CTXY-150002-6. Results will be published in peer-reviewed journals with deidentified data made available on FigShare.

Trial registration number: ChiCTR-ICR-15006053; Pre-results.

\section{Strengths and limitations of this study}

- The application of mobile health is designed not as a stand-alone technological solution but as a health system strengthening tool that serves to integrate the patient care provided by lay health supporters, village doctors, mental health administrators and psychiatrists.

- The active engagement of family members augments case supervision.

- The study, evaluating the real-world effectiveness of LEAN (Lay health supporter, e-platform, award, and iNtegration), emphasises the implementation parts so as to increase the likelihood of scaling up the potentially effective solution.

- The trial is intent on having global implications, especially insofar as the intervention is designed to exclude elements peculiar to China's socioeconomic and/or political situation.

- The short duration may not allow sufficient assessment of functional changes and limit analysis of the long-term effect on adherence.

- The choice of relatively simple assessment tools (pill counts vs urinalysis) may create challenges of obtaining an accurate adherence level.

- Assuming that improved medication adherence will lead to better patient life functioning may be problematic.

\section{INTRODUCTION}

Background and rationale

Schizophrenia, characterised by hallucination, delusion, disorganised thinking and negative symptoms, is a chronic and disabling mental disorder which is commonly associated with impairment in social and occupational functioning. ${ }^{1}$ Though schizophrenia cannot be cured, most people with schizophrenia can be effectively treated for symptoms with antipsychotic medicines. ${ }^{2}$ 
However, of the treated patients, $50 \%$ are non-adherent with medication; ${ }^{3}$ moreover, even under conditions of compliance, $50 \%$ of patients suffer relapse within 1 year of their latest episode. ${ }^{4}$ The ' 686 ' Program, a massive coutrywide government effort in China, is a relatively inexpensive and practical model that provides community-based mental healthcare with limited human and financial resources. ${ }^{5} 6$ However, the programme faces the challenges of poor medication adherence and high relapse- $26 \%$ of the programme participants never, $39 \%$ intermittently, and only $35 \%$ regularly take the prescribed medications. ${ }^{7}$ This research aims to develop and evaluate a financially and operationally feasible and sustainable intervention (with the acronym LEAN (Lay health supporter, e-platform, award, and iNtegration)) to address those ' 686 ' programme challenges.

\section{Hypothesis}

We hypothesise that the LEAN plus ' 686 ' solution, as compared to the present ' 686 ' standard of care only, will improve medication adherence, reduce the incidence of schizophrenia symptoms, and ultimately result in improved social and occupational functioning for enrollees.

\section{STUDY SETTING}

The intervention will be implemented and tested in '686' programme participants in the Xiang dialect area (a total of 9 towns) of the rural townships of Liuyang Municipality in the Hunan province of China, with an intent to produce solutions that can be adapted and applied in other low and middle income countries (LMCs) with limited mental health resources. Liuyang has developed a three-tier '686' model extending from Liuyang Mental Health Hospital (MHH) to township health centres to village clinics that consist of five components: (1) patient screening by village doctors (VDs) and mental health administrators (MHAs); (2) MHAs registering the confirmed cases to the "686" programme; (3) Psychiatrists touring townships to provide free consultation and medication every 2 months ('bi-monthly visits'); (4) case management by MHA; and (5) regular monitoring by $\mathrm{VDs}^{8-10}$ (figure 1). We should note that while Liuyang provides free antipsychotics to all its programme enrollees, in other parts of China, often only a subset of the programme participants receive free medication.

\section{LEAN}

LEAN as an acronym is somehow inspired by Toyota's principle in lean manufacturing, ${ }^{11}$ although our focus is on adding value, minimising waste, and maintaining simplicity throughout programme implementation. The acronym LEAN summarises the critical components of the proposed intervention (figure 2). The LEAN participants can opt out of LEAN anytime by texting us or by informing VDs, MHAs by phone or in person.

\section{Lay health supporter}

For each patient in the intervention, LEAN will identify an LHS-a member of the patient's family if possible or a community volunteer (such as a member of the village senior club) - who will perform simple but important roles in support of the patient: (1) facilitate patient medication adherence with prompts from the e-reminders, (2) monitor for early signs of relapse and for medication side effects using checklists from the e-monitor, and (3) team up with the VD and the township MHA to facilitate treatment adjustments and, if needed, emergent hospital care.

\section{E-platform}

The e-platform employs three main modules: The e-reminder sends the patient up to two reminders either by text or voice messages at $15 \mathrm{~min}$ interval until the patient responds with confirmation that the scheduled medication has been taken. Failure to send a confirmation will trigger up to two text alerts to the patient's LHS, prompting the LHS to check in with the patient and text back the result. The e-monitor assists LHSs and patients in detecting signs of relapse and monitoring medication side effects using relevant checklists texted to the patient and LHS at regular intervals (see relapse checklist in the online supplementary appendix). Finally, the e-educator will send periodic SMS messages to the patient, LHS, MHA and VD to educate them on schizophrenia symptoms, medication, adherence strategies, relapse, rehabilitation and social resources.

\section{Award system}

Patients and LHSs will accumulate points for responding to SMS messages. Each of their texted confirmations back to the LEAN system will accumulate one point, which will be recorded automatically by the computer system. The points, counted every 2 months, will advance their Taekwondo-like belt ranking and entitle them to a small gift of US\$2-3 such as soap bars when they come for the bimonthly visit to be presented by a LEAN programme staff member.

\section{iNtegration}

The efforts of the patient and LHS to improve medication adherence and reduce relapses will be integrated and facilitated by the e-platform with those of the VD, MHA and psychiatrist so that the innovations of LEAN strengthen the existing health system. With this integration, non-adherence and relapses detected can then be actually handled with LHSs, VDs, MHAs and psychiatrists taking concerted effort for prompt treatment adjustments or referrals for emergent hospitalisation.

\section{Mechanism of LEAN}

The mechanism of LEAN medication adherence is based on an adapted health belief model (HBM) (figure 3). ${ }^{12}{ }^{13}$ According to this theory, people with schizophrenia make their medication adherence 


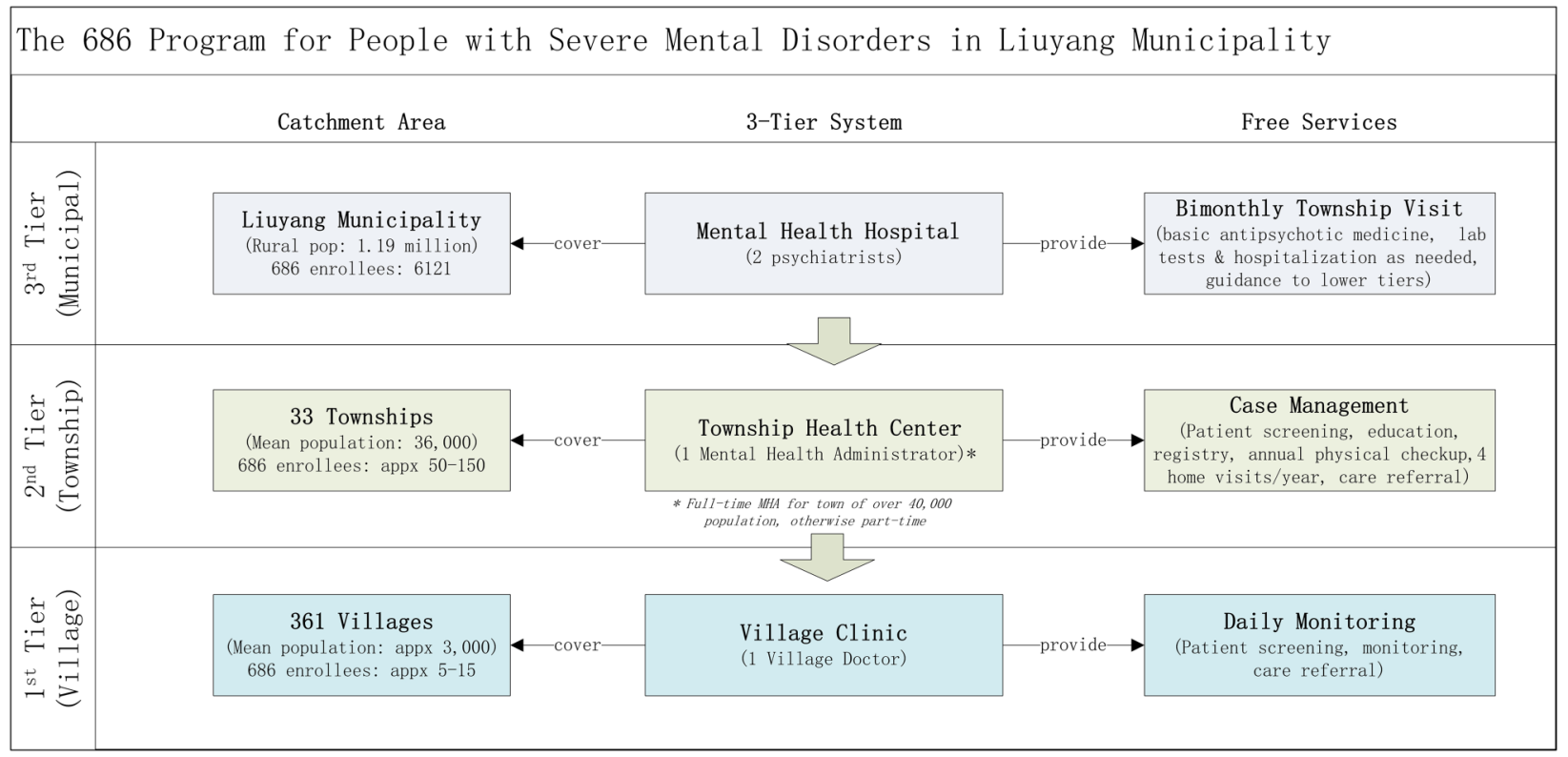

Figure 1 The '686' Program Service Model.

decisions based on push (patients' self-motivation in improving health) and pull factors that include three elements: (1) patients' perception of the threat posed by schizophrenia; (2) patients' perceived net benefit of adhering to therapy, a calculation involving the benefits of therapy minus costs; and (3) action cues such as the aforementioned e-reminders or mass media health promotion campaigns. Figure 3 illustrates the interface of various LEAN elements with the components of the HBM.
The development of LEAN has been guided by the HBM as a theoretical framework, as well as informed by empirical evidence, particularly in the areas of human resources for health (HRH) and mobile health (mHealth). Much of the literature in HRH suggests that 'task shifting'-cascading appropriate tasks from more skilled psychiatrists to less specialised MHAs/VDs and to LHSs-improves access and efficacy when HRH are lacking or deficient ${ }^{14}{ }^{15}$ (Liuyang has only 1.35 psychiatrists/1.42 specialist nurses vs 8.59 psychiatrists/29.15

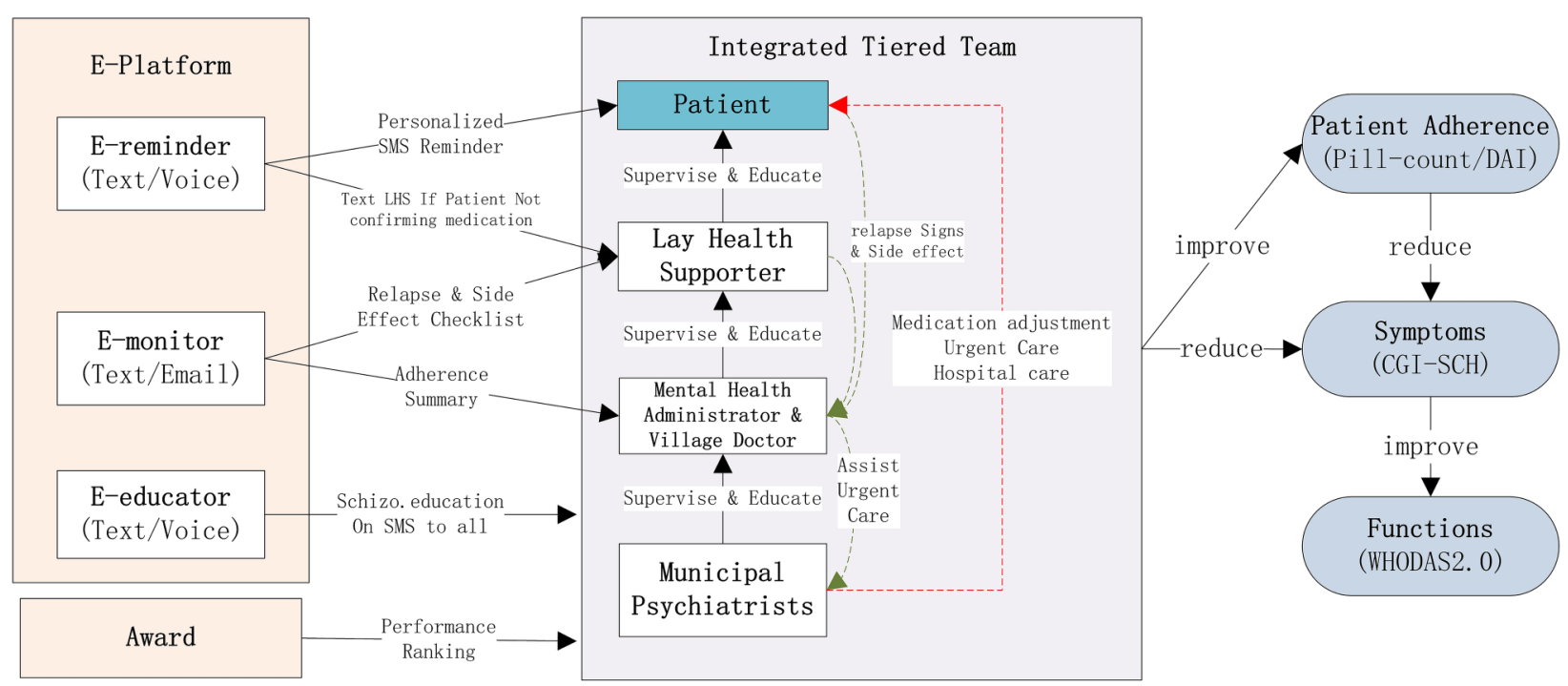

iNtegrated as the LEAN solution

Figure 2 LEAN. Lay health supporter (LHS). E-platform with e-reminder, e-monitor and e-educator via mobile text/voice messaging. Award system analogous to Taekwondo ranks. iNtegrating the $L, E$ and $A$ and ' 686 ' Program structure into a lean and coordinated approach. DAI, Drug Attitude Inventory-10; WHODAS, WHO Disability Assessment Schedule. 


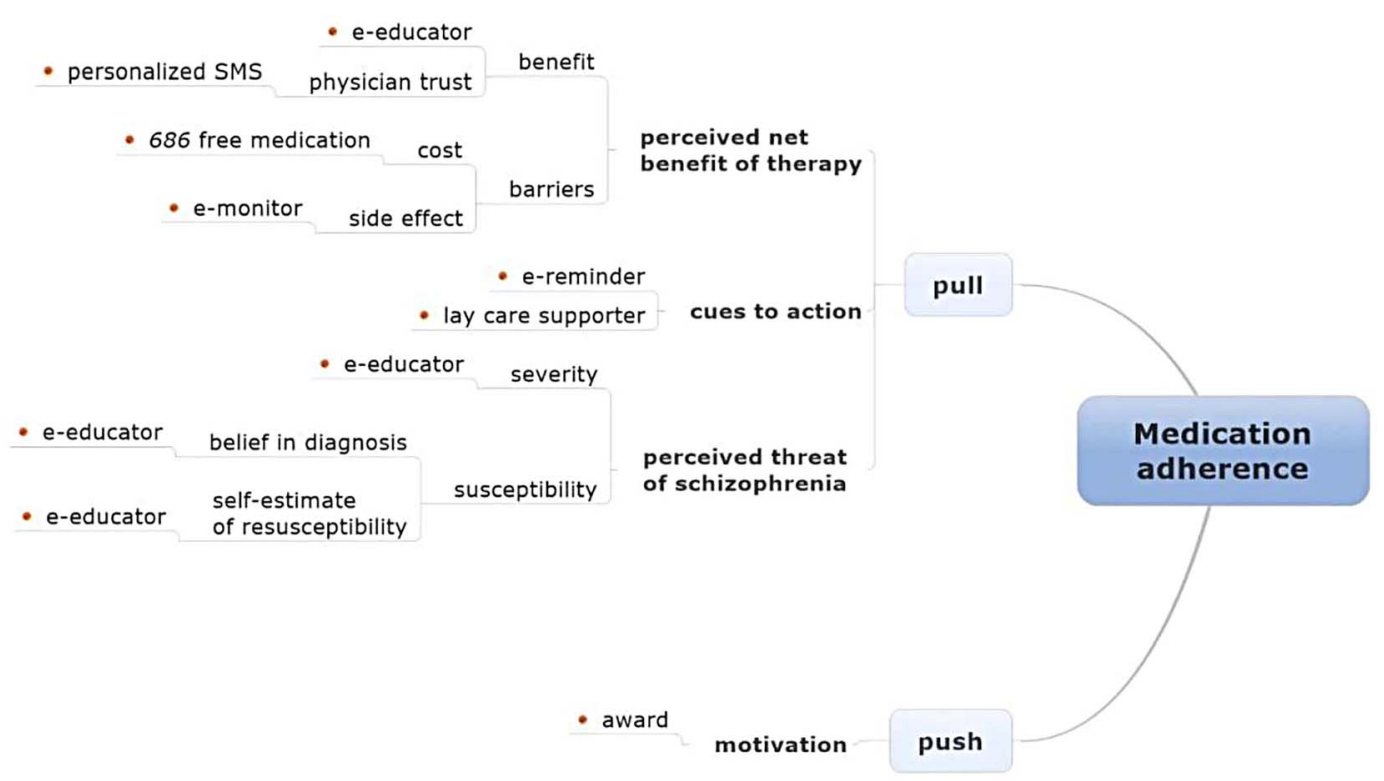

Figure 3 Mechanism for lean medication adherence. Note: The red dots indicate LEAN components. Source: adapted from the health belief model.

nurses for high-income countries per 100000 population in 2011). The e-platform facilitates efficient communication and integration of this network of human resources. Moreover, much evidence supports the use of reminders to improve medication adherence. ${ }^{16-20}$

\section{STUDY POPULATION AND THE LEAN SAMPLE}

People in Liuyang speak three distinct dialects: Gan, Xiang and Hakka. The Xiang dialect area, located in the west of Liuyang municipality, has 9 townships, 98 villages and a population of 356900 . The '686' Program maintains a roster of patients with schizophrenia in the Xiang dialect area of Liuyang municipality (total: 631 in 2011) (figure 4), which forms the study population. The characteristics of this population, most relevant to our study, are summarised in table 1 . The Xiang dialect population is selected due to (1) the efficiency to recruit, train and collect data in a more focused population; (2) the Xiang dialect group being the majority group in Hunan province while the other two dialect groups in Liuyang are historically immigrants from other provinces; and (3) the long and rich past research experience of our group in this area that provides additional data and information for the LEAN study, such as educational levels of all MHAs.

\section{Inclusion and exclusion criteria}

The following criteria more precisely define the study population by establishing eligibility requirements for
Figure 4 Map of the Xiang Dialect area of Liuyang. Note: The yellow-shaded region on the map of China is Hunan Province.

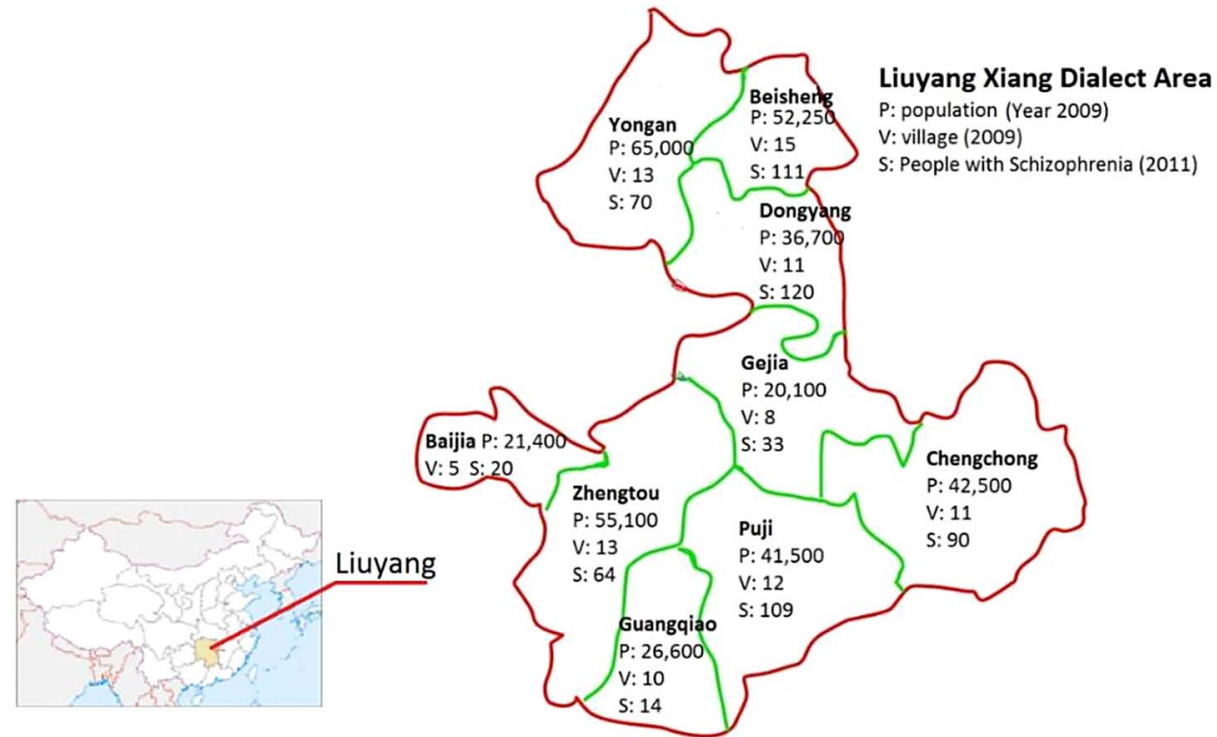




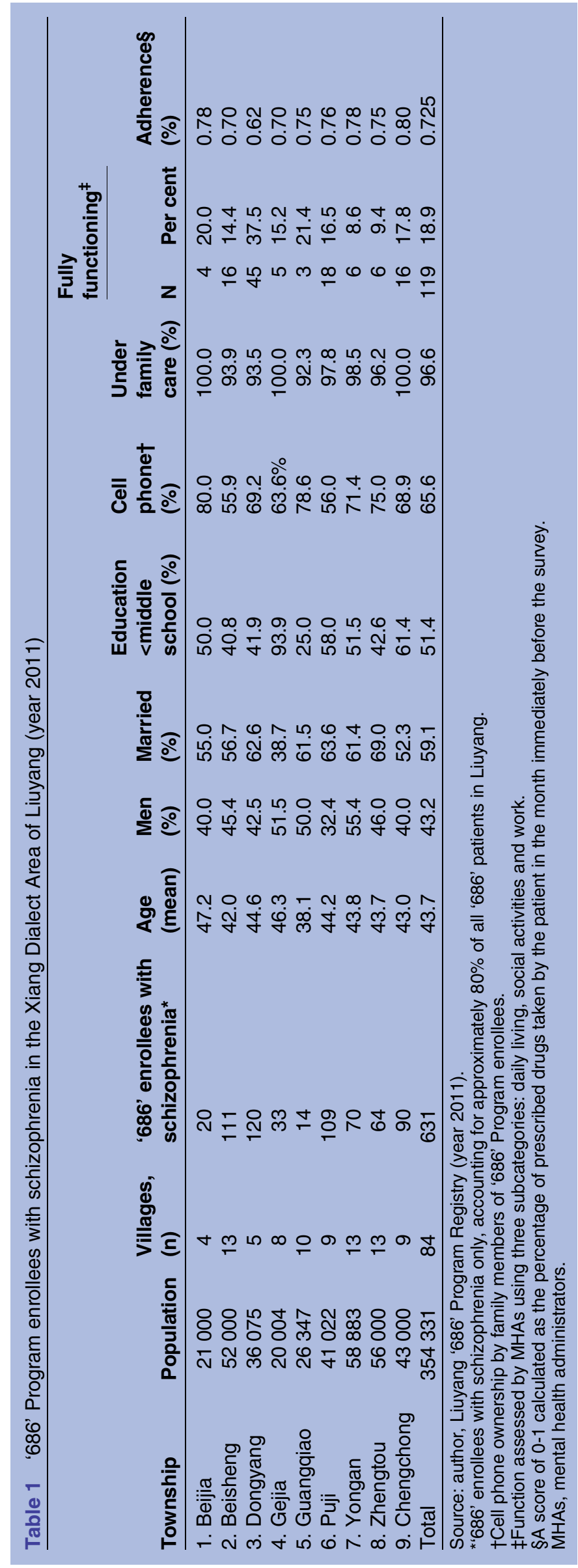

subject recruitment. Since villagers and LHSs without a phone will be given a free basic phone and subscription plan, the phone ownership is not included in the inclusion or exclusion criteria. Rationales for inclusion and exclusion criteria are given in parentheses.

\section{Inclusion}

1. '686' Program enrollees.

2. Diagnosed as having schizophrenia according to criteria established in the Diagnostic and Statistical Manual of Mental Disorders-5 (DSM-5) $)^{21}$

3. Physically reside in the Xiang dialect area of Liuyang Municipality.

\section{Exclusion}

1. Individuals registered in the Xiang dialect area of Liuyang Municipality, but living elsewhere as migrant workers (as a community-based intervention, LEAN requires residence in the local community)

2. Patients who have missed three immediate consecutive past drug refills (in this case, they have de facto dropped out of the ' 686 ' Program)

3. People who are currently hospitalised (again, LEAN intervention requires sustained community residence)

4. People physically incapable of using voice or text messaging, for example, individuals with hearing and/or vision impairment, or who are severely disabled (ability to utilise SMS is necessary for the LEAN intervention).

\section{Sampling frame, the LEAN sample and recruitment}

The most recent ' 686 ' Program registry of patients with schizophrenia will be used as the sampling frame, from which we aim to draw 258 patients as the LEAN sample. To that end, a statistician otherwise not associated with the project will first create a recruitment list of 400 people drawn at random from the sampling frame. Assuming that $15 \%$ of those selected will prove ineligible and that a further $20 \%$ will elect not to participate, an initial list of 400 should ensure a final recruitment of no less than 258 participants. MHAs will provide an initial screening by cross-checking the recruitment list against their own records in order to verify eligibility. Recruitment by project staff will occur during patients' bimonthly medication refill visits, when psychiatrists will reconfirm the diagnoses of those on the list. Project staff will conduct home visits within 1 month of their expected bimonthly visit to recruit those not contacted at the refill visits. At the end of the recruitment, the LEAN sample will be randomly divided by the same statistician into a treatment group and a control group of equal sizes by a statistician not otherwise involved in the study (figure 5).

\section{Sample size calculation}

Though the distribution of our primary outcome (adherence, scored as the percentage of drugs taken of 


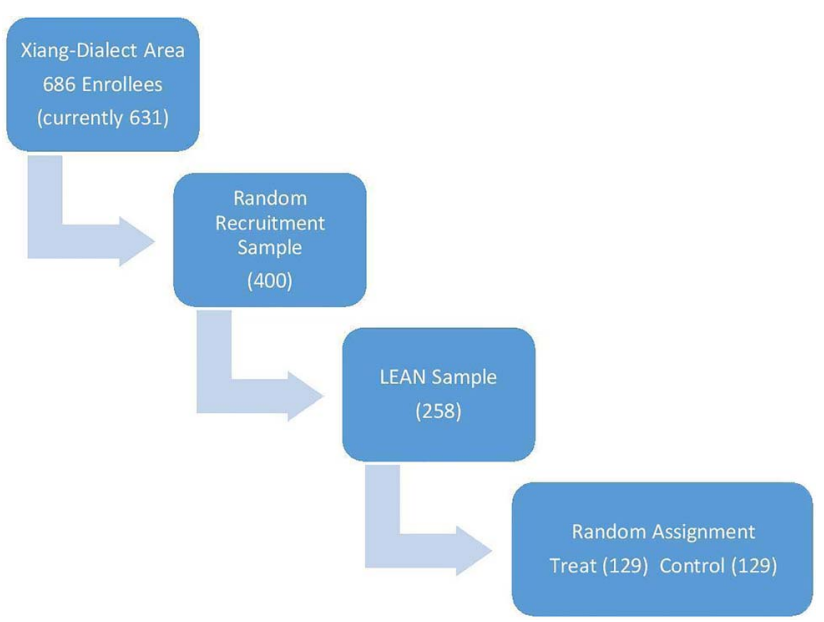

Figure 5 the lean population, sample and assignment.

those prescribed) is unlikely to be normally distributed, the sample calculation follows standard procedures for the hypothesis of equal population means based on $\mathrm{t}$ test and the comparison of sample means. Since our sample size is large, the central limit theorem ensures that our sample means will be approximately normally distributed, regardless of the underlying distribution of the data.

Assuming a 5\% type I error and a $10 \%$ dropout ratio for a total sample size of 258 (129 for each of the two comparison groups), the study of 232 participants (after $10 \%$ dropping out of 258) will have $85 \%$ power to detect an effect size of 0.13 (see online supplementary material appendices). This means that if the adherence score for the control group is $0.72(\mathrm{SD}=0.33)$, the study will have sufficient power to detect a programme effect if adherence for the treatment group is equal to or greater than 0.85 . The control adherence of 0.72 used in the sample calculation is based on the self-reported adherence of 0.75 in our study population from the ' 686 ' registry.

The proposed sample size of 258 will also satisfy the power requirement for a subgroup analysis of patients who are non-adherent at baseline. Given the ratio of non-adherence to full adherence $(0.55: 0.45)$ of the population reported in the registry, the study will include at least 140 baseline non-adherent participants available for the subgroup analysis. Again, assuming 5\% type I error and a $10 \%$ dropout rate, the study will have $85 \%$ power to detect an effect size of 0.18 among the subgroup: if the adherence rate for the control is 0.42 $(\mathrm{SD}=0.35)$, the study will be powered to detect a programme effect if the adherence of the treatment group is equal to or greater than 0.6 (table 2).

\section{METRICS AND MEASUREMENT}

Primary and secondary outcomes

The primary outcome will be a continuous medication adherence score from 0 (no adherence) to 1 (complete adherence), calculated as the percentage of drugs taken out of those prescribed over a designated time period (the preceding month). Medication adherence was chosen as the primary outcome on the grounds that (1) adherence correlates with symptom relief, and symptoms correlate with function; $;^{22} 23$ (2) significant improvement in symptoms and functions is likely to extend beyond the duration of the study; and (3) improving adherence is valuable in its own right. However, symptoms and functions will also be tracked as the secondary outcomes.

\section{Methods of assessment and timeline}

Figure 6 summarises how and when we assess outcomes, which piggyback on '686' Program activities, in particular, the bimonthly meetings with patients. All data will be double-entered into and managed by the Research electronic data capture (REDCap) system. ${ }^{24}$ All outcome assessors, including psychiatrists and programme staff, will be blinded to the control or treatment status of programme participants; any inadvertent unblinding will be noted in order to record the time of the incident and persons involved.

\section{Medication adherence: pill counts}

Pill counts, to be conducted by project staff when patients bring their pill bottles to the bimonthly refill, will be used as the primary, objective and inexpensive measurement of medication adherence, to be complemented by pharmacy dispensing records from the ' 686 ' registry system. Other objective measures, such as the serum/urine drug level, ${ }^{25}$ are clinically and financially impossible to implement. In addition, the Morisky Medication Adherence Scale, ${ }^{26}$ the Brief Adherence Rating Scale (BARS) ${ }^{27}$ and the Drug Attitude Inventory-10 (DAI-10) ${ }^{28}$ will supplement the objective assessment. At baseline and again at the end of the study, patients who were no-shows at the bimonthly visit will be visited and assessed at their homes.

\section{Symptoms: Clinical Global Impression in Schizophrenia}

From among the 'big three' instruments for schizophrenic symptoms, ${ }^{29}$ we chose the Clinical Global Impression in Schizophrenia (CGI-Sch) primarily due to its brevity and ease of use. ${ }^{30}$ ' 686 ' Program psychiatrists will assess patients using the CGI-Sch during bimonthly visits throughout the trial.

\section{Functions: WHODAS 2.0}

LEAN will use the 12-item proxy-administered WHO Disability Assessment Schedule 2.0 to assess patient functions. We select this instrument because of its brevity to administer, excellent psychometric properties and availability of a validated Chinese version. ${ }^{31}{ }^{32}$ Public health students enlisted as programme staff will administer the WHODAS to patients and their family members during bimonthly visits. 
Table 2 sample size calculation scenarios

\begin{tabular}{|c|c|c|c|c|c|}
\hline & \multicolumn{2}{|c|}{ Adherence score } & \multicolumn{2}{|c|}{ Sample size needed* } & \multirow[b]{2}{*}{ Total } \\
\hline & Control & Treat & Control & Treat & \\
\hline LEAN Sample & $0.72(0.33) \dagger$ & $0.85(0.33)$ & 129 & 129 & $258 \ddagger$ \\
\hline Non-adherent subgroup§ & $0.42(0.35)$ & $0.60(0.35)$ & 70 & 70 & 140 \\
\hline
\end{tabular}

\section{Other measures}

Since a side effect of anti-antipsychotics may relate to adherence, the brief and self-implemented Glasgow Antipsychotic Side-effect Scale (GASS) will be used to generate a side-effect score. ${ }^{33} \mathrm{~A}$ few other 'public health' indicators such as suicide, drug abuse, attacking people, destroying things and wandering will be captured by the existing ' 686 ' registry. In addition, many process, cost and service utilisation indicators will be captured and recorded by the e-platform logs and ' 686 ' administrative registry. These process indicators will facilitate analysis of various links in the LEAN mechanism, and surveillance for breaks in the chain.

\section{TRIAL DESIGN}

We adopt a wait-list design with participants followed up for 6 months after launch of the intervention. The waitlist control design is increasingly used in psychotherapy studies, primarily to address the ethical dilemma involved in withholding a potentially beneficial treatment from the control group. Participants recruited into the study are randomised into a treatment group and a 'wait-listed' control group. In stage 1 (the 6-month period following programme initiation), the intervention will be applied to the intervention group only, while the wait-list group will receive usual care per the regular '686' protocol; in stage 2 (a subsequent 6-month period), the wait-list group will receive the intervention, having 'waited' through stage one. Analysis of the intervention will be conducted on the basis of the baseline and end point data collected on both groups during stage one only due to our budget constraints on data collection. Consequently, the only difference between a wait-list design and a traditional two-arm randomised control trial (RCT) is that the control group is also able to benefit from the treatment once the formal study is complete.

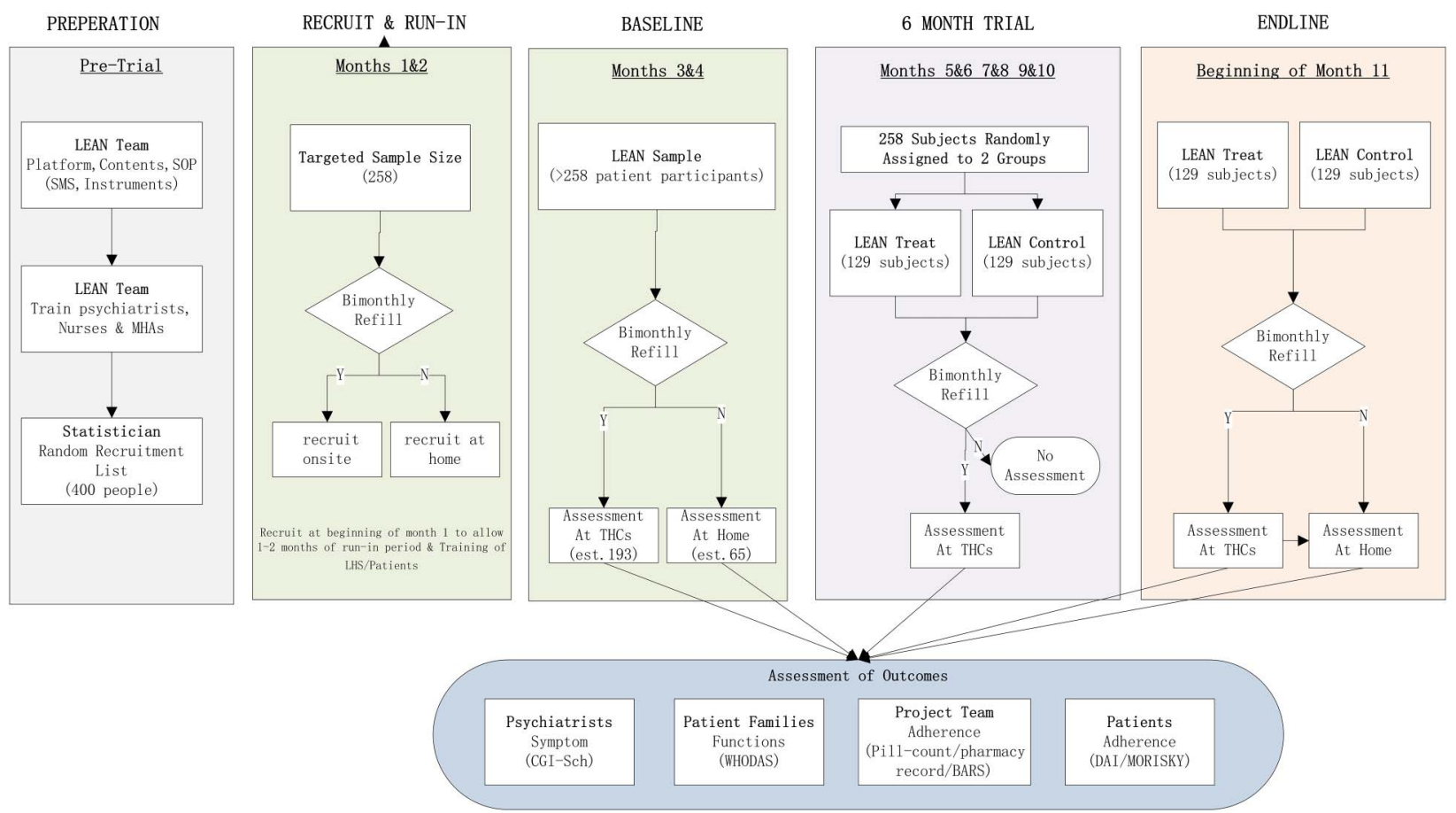

Figure 6 Recruitment and outcome assessment. BARS, Brief Adherence Rating Scale; CGI-Sch, Clinical Global Impression in Schizophrenia; DAI, Drug Attitude Inventory-10; Morisky, Morisky Medication adherence Scale; SOP, standard operation procedures; THCs, township health centres; WHODAS, WHO Disability Assessment Schedule. 


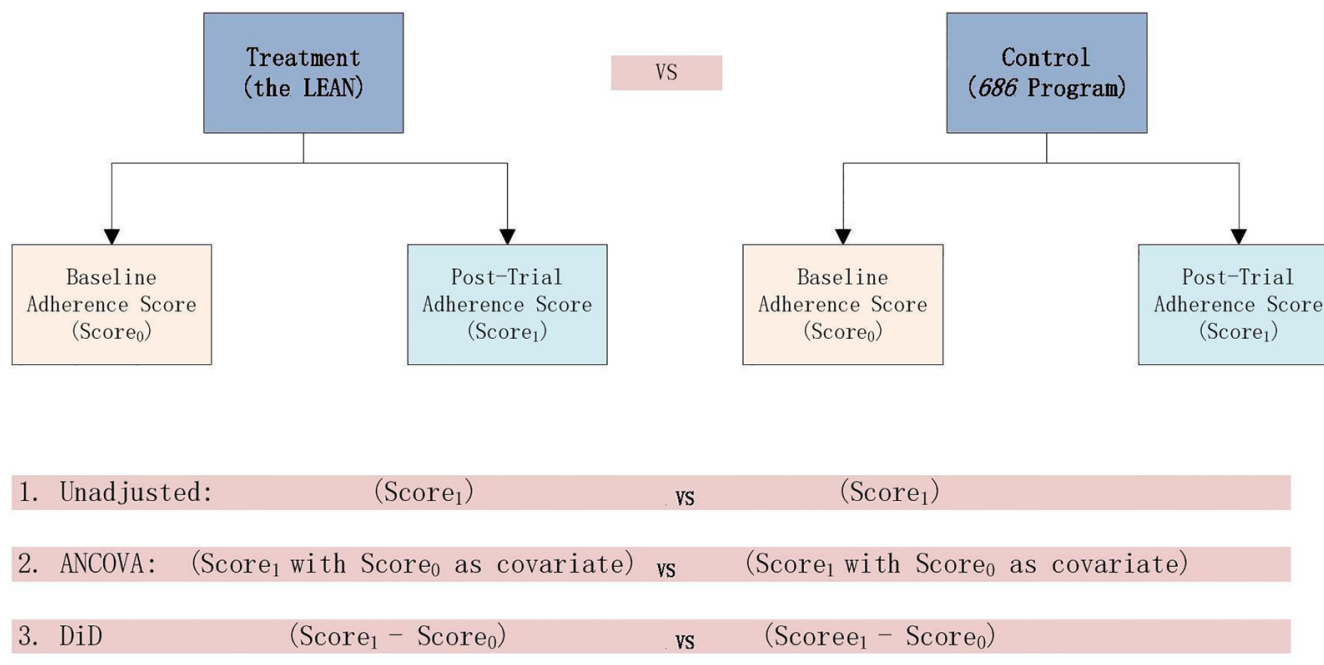

Figure 7 Three approaches to RCT analysis. Source: adapted from Siyuan Zhang's paper. ${ }^{34}$ ANCOVA, analysis of covariance; DiD, difference-in-difference; RCT, randomised controlled trial.

\section{MODEL AND ANALYSIS}

Unadjusted analysis, analysis of covariance and difference-in-difference

We mainly considered the issue of efficiency (precision of the estimator) and bias in our choice of analytical methods. The literature suggests that analysis of covariance provides higher efficiency than differencein-difference and the unadjusted model in RCT and is the optimal model for RCT analysis ${ }^{34}$ (figure 7 ). The LEAN analysis will include as covariates the strong baseline predictors of outcome that are empirically suggested by other studies, and will comprise adherence, WHODAS and CGI-Sch scores, as well as indices of negative symptoms, substance use, medication side effects and family supervision. ${ }^{35}$ It should be noted that while our response variable, expressed as an adherence score from 0 to 1 , may yield values greater than one, those out-of-bound predictions do not invalidate the model since the study's purpose is to produce a 'risk difference' (difference in mean adherence between intervention and control groups) rather than an individual prediction. Critically, the large sample size and the central limit theorem ensure that this approach will yield valid inferences of the risk difference despite non-normal adherence outcomes.

\section{Intent-to-treat}

An intent-to-treat (IIT) analysis will be used to analyse all participants regardless of the treatment actually received. Estimating the IIT effect is more appropriate than the per-protocol or per-treat methods since the LEAN trial is a pragmatic trial, which is to say, it is meant to determine the effectiveness of LEAN as a realworld solution.

\section{Subgroup analysis}

We plan to conduct two subgroup analyses, both with a strong theory base and possible interaction effects. The first concerning the non-adherent group at baseline is sufficiently powered (table 2) (our adherence-focused intervention is more likely to work better for the initially non-adherent group). The other subgroup analyses will be conducted to assess level of functions.

\section{Missing data}

Reasons for missing data will be recorded. Multiple imputation methods will be used so that sensitivity analyses will be conducted to assess the robustness of trial results under different methods.

\section{MONITORING}

Considering the short duration of the intervention, we do not have a data monitoring committee. At the midpoint of the trial, outcomes and text messaging data will be analysed to detect any abnormality. The text messaging system also provides a means for ongoing monitoring of any patient response.

\section{ETHICS AND DISSEMINATION}

The study has obtained institution review board (IRB) approval from the University of Washington (49464 G) and Central South University (CTXY-150002-6). Any substantive modification to the protocol will seek a formal approval from the IRBs. Programme staff will train and obtain informed consent from both patients and LHSs. Patient data will be securely entered and stored in RedCap and only deidentified information will be used for analysis. Study results will seek peer-reviewed publications with deidentified data made available on Figshare. ${ }^{36}$

\section{DISCUSSION}

Several aspects of this study are worth noting. First, the application of mHealth is designed not as a standalone technological solution but as a health system strengthening tool that serves to integrate the patient care provided by lay health supporters (LHS), VDs, MHAs and psychiatrists. 
Second, the active engagement of LHS augments case supervision. Third, the study, evaluating the real-world effectiveness of LEAN, emphasises the implementation parts so as to increase the likelihood of adopting the potentially effective solution. Fourth, the trial is intent on having global implications, especially insofar as the intervention is designed to exclude elements peculiar to China's socioeconomic and/or political situation.

The study is faced with several limitations. First, its short duration may not allow sufficient assessment of functional changes and limit analysis of the long-term effect on adherence. Second, our choice of relatively simple assessment tools (pill counts vs urinalysis) may create challenges of obtaining accurate adherence levels. Third, assuming that improved medication adherence will lead to better patient life functioning may be problematic. There is concern that psychiatrists with limited training from Liuyang $\mathrm{MHH}$ may deliver inappropriate treatments, adherence to which will be of insufficient benefit. Finally, despite efforts to ensure the generalisability of LEAN, the existing ' 686 ' infrastructure (particularly the availability of free basic antipsychotics and the bimonthly physiatrists' visit) may make Liuyang a unique location even within China. We hope that the spirit of LEAN will provide useful information for other LMCs. For instance, LEAN may be adapted to manage patients discharged from mental facilities who continue to take free or paid medications.

\section{Author affiliations}

${ }^{1}$ Sun Yat-sen Global Health Institute (SGHI), School of Public Health, Sun Yatsen University, Guangzhou, Guangdong, China

${ }^{2}$ Department of Global Health, University of Washington, Seattle, Washington, USA

${ }^{3}$ Xiangya School of Public Health, Central South University, Changsha, Hunan, China

${ }^{4}$ Department of Psychiatry, University of Rochester Medical Center, Rochester, New York, USA

${ }^{5}$ Department of Biostatistics, University of Washington, Seattle, Washington, USA

${ }^{6}$ Institute for Health Metrics and Evaluation, University of Washington, Seattle, Washington, USA

${ }^{7}$ Department of Phycology, University of Washington, Seattle, Washington, USA

${ }^{8}$ Department of Epidemiology, School of Public Health and Tropical Medicine, Tulane University, New Orleans, Louisiana, USA

${ }^{9}$ Division of Health Promotion and Behavioral Sciences, University of Texas Health Science Center School of Public Health at Houston, Houston, Texas, USA

${ }^{10}$ Austin Regional Campus Michael \& Susan Dell Center for Healthy Living, UTHealth School of Public Health, Austin, Texas, USA

Acknowledgements The authors thank our team members for their critical contribution to the implementation of this project including our project managers Juan Nie at SYSU and Yunfang Wang at CSU, who contributed critically to the IRB reviews.

Contributors All authors contributed to the conceptualisation and the design of the study. WG obtained a majority of the funding. DX and WG conceived of the prototype of the intervention, the study design, analytical methods and creation of the team. DX drafted the first manuscript. SX and WG secured the study site. EDC and SX contributed significantly to the intervention strategy and the methods of outcome assessment. JPH, MN and HH provided critical review and revision to the design and analytical methods of the study. JS contributed to the theoretical framework of the study. KLS edited and improved the manuscript. HSB helped design and write the economic evaluation part of the protocol. SG steered the direction of the study and contributed significantly to the revision of the manuscript. All authors read and revised the initial manuscript and approved the final version.

Funding The authors acknowledge the generous support by the China Medical Board through its Health Policy and Systems Sciences Open Competition grant (grant number 12-114, WG, PI). The project receives additional research funding from NIH research training grant \# R25 TW009345 funded by the Fogarty International Center (DX, Fogarty fellowship). WG and EDC were supported, in part, by NIH grant D43 TW009101 (EDC, PI) as well.

Competing interests None declared.

Ethics approval University of Washington in US (49464 G); Central South University in China (CTXY-150002-6).

Provenance and peer review Not commissioned; externally peer reviewed.

Data sharing statement We will make our data publicly available through FigShare after the main studies are published in peer-reviewed journals

Open Access This is an Open Access article distributed in accordance with the Creative Commons Attribution Non Commercial (CC BY-NC 4.0) license, which permits others to distribute, remix, adapt, build upon this work noncommercially, and license their derivative works on different terms, provided the original work is properly cited and the use is non-commercial. See: http:// creativecommons.org/licenses/by-nc/4.0/

\section{REFERENCES}

1. American Psychiatric Association. Diagnostic and statistical manual of mental disorders fifth edition (DSM-5). Arlington, VA: American Psychiatric Association, 2013.

2. Leucht S, Cipriani A, Spineli L, et al. Comparative efficacy and tolerability of 15 antipsychotic drugs in schizophrenia: a multiple-treatments meta-analysis. Lancet 2013;382:951-62.

3. Babiker IE. Noncompliance in schizophrenia. Psychiatr Dev 1985;4:329-37.

4. Vaughn CE, Snyder KS, Jones $S$, et al. Family factors in schizophrenic relapse: replication in California of British research on expressed emotion. Arch Gen Psychiatry 1984;41:1169-77.

5. Good BJ, Good MJ. . Significance of the 686 Program for China and for global mental health. Shanghai Arch Psychiatry 2012;24:175-7.

6. Ma H. Integration of hospital and community services-the '686 Project'-is a crucial component in the reform of China's mental health services. Shanghai Arch Psychiatry 2012;24:172-4.

7. Li Q, Huang X, Wen $\mathrm{H}$, et al. Retrospective analysis of treatment effectiveness among patients in Mianyang Municipality enrolled in the national community management program for schizophrenia. Shanghai Arch Psychiatry 2012;24:131-9.

8. Changsha government. "Working procedures of the program for the management of people with Severe Mental disorders in Changsha Municipality (interim)." http://www.changsha.gov.cn. Changsha Government Web Portal, 16 November 2014. Web. 16 November 2014. http://goo.gl/KLX2Ul

9. Dongming $\mathrm{S}$, Jiyue $\mathrm{H}$, Wenjun $\mathrm{Z}$, et al. The development of community based mental health services in Liuyang. Chinese Mental Health J 2011;25:517-20.

10. "Working plan of Mental Health 2012." 2012 mental health working plan. Web. 05 December 2014. http://www.liuyang.gov.cn/lyswszf/ xxgkml/zfgzbmxxgkml/swsj/ghjh/gzjh/ndjh/content_78177.html

11. Weinstock D. Lean healthcare. J Med Pract Manage 2008;23:339-41.

12. Becker $\mathrm{MH}$, Maiman LA. Sociobehavioral determinants of compliance with health and medical care recommendations. Med Care 1975;13:10-24.

13. Rosenstock IM. Why people use health services. Milbank Mem Fund Q 1966;44:(Suppl):94-127.

14. Simpson EL, House AO. Involving users in the delivery and evaluation of mental health services: systematic review. $B M J$ 2002;325:1265.

15. Mutamba BB, van Ginneken N, Smith Paintain L, et al. Roles and effectiveness of lay community health workers in the prevention of mental, neurological and substance use disorders in low and middle income countries: a systematic review. BMC Health Serv Res 2013;13:412. 
16. Zygmunt A, Olfson M, Boyer CA, et al. Interventions to improve medication adherence in schizophrenia. Am J Psychiatry 2002;159:1653-64.

17. van Dulmen $S$, Sluijs $E$, van Dijk $L$, et al. Patient adherence to medical treatment: a review of reviews. BMC Health Serv Res 2007;7:55

18. Dixon LB, Dickerson F, Bellack AS, et al, Schizophrenia Patient Outcomes Research Team (PORT). The 2009 schizophrenia PORT psychosocial treatment recommendations and summary statements. Schizophr Bull 2010;36:48-70.

19. Pekkala E, Merinder L. Psychoeducation for schizophrenia Cochrane Database Syst Rev 2002;(2):CD002831.

20. Lincoln TM, Wilhelm K, Nestoriuc Y. Effectiveness of psychoeducation for relapse, symptoms, knowledge, adherence and functioning in psychotic disorders: a meta-analysis. Schizophr Res 2007:96:232-45.

21. American Psychiatric Association. Diagnostic and statistical manual of mental disorders, (DSM-5®). American Psychiatric Pub, 2013.

22. Burns T. Evolution of outcome measures in schizophrenia. $\mathrm{Br} J$ Psychiatry 2007;191:s1-6.

23. Eack SM, Newhill CE. Psychiatric symptoms and quality of life in schizophrenia: a meta-analysis. Schizophr Bull 2007;33:1225-37.

24. Harris PA, Taylor R, Thielke R, et al. Research electronic data capture (REDCap) - a metadata-driven methodology and workflow process for providing translational research informatics support. J Biomed Inform 2009;42:377-81.

25. Pomykacz B, Mao M, Weiss RD, et al. A review of brief medication-adherence instruments used in patients with schizophrenia and bipolar disorder. Harv Rev Psychiatry 2007;15:259-63.

26. Morisky DE, Green LW, Levine DM. Concurrent and predictive validity of a self-reported measure of medication adherence. Med Care 1986;24:67-74.
27. Byerly MJ, Nakonezny PA, Rush AJ. The Brief Adherence Rating Scale (BARS) validated against electronic monitoring in assessing the antipsychotic medication adherence of outpatients with schizophrenia and schizoaffective disorder. Schizophr Res 2008;100:60-9.

28. Hogan TP, Awad AG, Eastwood R. A self-report scale predictive of drug compliance in schizophrenics: reliability and discriminative validity. Psychol Med 1983;13:177-83.

29. Mortimer AM. Symptom rating scales and outcome in schizophrenia. Br J Psychiatry Suppl 2007;191:s7-s14.

30. Haro JM, Kamath SA, Ochoa S, et al. The Clinical Global Impression-Schizophrenia scale: a simple instrument to measure the diversity of symptoms present in schizophrenia. Acta Psychiatr Scand Supp/ 2003;107:16-23.

31. Üstün TB, ed. Measuring health and disability: manual for WHO disability assessment schedule WHODAS 2.0. World Health Organization, 2010.

32. Üstün TB, Chatterii S, Kostanjsek N, et al. Developing the World Health Organization disability assessment schedule 2.0. Bull World Health Organ 2010;88:815-23.

33. Waddell L, Taylor M. A new self-rating scale for detecting atypical or second generation antipsychotic side effects. J Psychopharmacol 2008:22:238-43.

34. Zhang S, Paul J, Nantha-Aree M, et al. Empirical comparison of four baseline covariate adjustment methods in analysis of continuous outcomes in randomized controlled trials. Clin Epidemiol 2014;6:227-35.

35. Fenton WS, Blyler CR, Heinssen RK. Determinants of medication compliance in schizophrenia: empirical and clinical findings. Schizophr Bull1997;23:637-51.

36. "Figshare." Web. 27 September 2015. http://figshare.com/about 